\title{
An efficient multiband envelope function approximation method for spintronics
}

\author{
X Cartoixà ${ }^{1}$, D Z-Y Ting ${ }^{2}$ and T C McGill ${ }^{1,3}$ \\ ${ }^{1}$ T J Watson, Sr, Laboratories of Applied Physics 128-95, California Institute of Technology, \\ Pasadena, CA 91125, USA \\ ${ }^{2}$ Jet Propulsion Laboratory, California Institute of Technology, Pasadena, CA 91109, USA \\ E-mail: tcm@ssdp.caltech.edu
}

Received 4 October 2002, in final form 17 December 2002

Published 23 January 2003

Online at stacks.iop.org/Nano/14/308

\begin{abstract}
We have developed an eight-band finite-difference envelope function approximation model capable of reproducing in almost all situations the true $\mathrm{D}_{2 \mathrm{~d}}$ or $\mathrm{C}_{2 \mathrm{v}}$ symmetry of [001] grown zinc-blende heterostructures. We have used our model to study the relative contributions of the bulk inversion asymmetry (BIA) and structural inversion asymmetry to the spin splitting in the conduction band of asymmetric AlSb/GaSb/InAs/AlSb quantum wells, and clarify apparently contradictory statements about the relative magnitude of the two contributions. We show that, in the system under study, the inclusion of BIA effects changes considerably the angular dependence and the magnitude of the splitting. We also investigate how BIA changes the transmission properties of a resonant tunnelling structure.
\end{abstract}

\section{Introduction}

In recent years, interest in developing spin-sensitive devices (spintronics) [1] has fuelled renewed investigations into spin phenomena in semiconductors. The aim is to control not only the spatial degrees of freedom of the electron, but also the spin degree of freedom. Useful spintronic devices can be devised if such control is attained. A number of such devices have already been proposed [2,3], including one suggested by the authors that could achieve high degrees of spin injection without the presence of external magnetic fields and just using nonmagnetic III-V semiconductors [4].

On one hand, the full understanding of the operation of spintronic devices will require having at one's disposal electron band structures where all spin effects are included; in much the same way as thorough knowledge of the 'usual' (meaning spin degenerate at each point in a reciprocal space) band structure is required to fully understand electronic devices. On the other hand, semiconductor heterostructures have been extensively used to create new classes of materials with engineered electronic properties, and they show promise for serving the same purpose for the spin properties. To meet these two demands, we present an improved eight-band envelope

3 Author to whom any correspondence should be addressed. function approximation (EFA) implementation that provides realistic band structure at the spin level for zinc-blende-based [001] superlattices and quantum wells (QWs), as well as an efficient method for computing spin-dependent tunnelling properties. As a consequence, the relative contribution of structural inversion asymmetry (SIA) versus bulk inversion asymmetry (BIA) to the spin splitting in the conduction subbands can be delineated. Thus the apparently contradictory statements of Lommer et al [5], stating that BIA (SIA) dominates the splitting in large-gap (narrow-gap) materials, and Cardona et al [6], listing a proportionality constant for the $k^{3}$-splitting [7] that roughly gets larger with smaller band gap, can be put in perspective.

Most of the standard implementations of the EFA for III-V heterostructures do not take into account the BIA present [7] in zinc-blendes [8, 9], and therefore do not include a potentially important source of spin splitting. Previously, only Rössler, Winkler et al $[10,11]$ have used an eight-band model with BIA. They compared their calculations to experimentally measured spin splittings [11]. However, in their work they did not study the symmetry properties of the heterostructure Hamiltonian or show that the eight-band model can reproduce the results of the two-band Hamiltonians close to the zone centre. 


\section{Methods}

There are several published eight-band $k \cdot p$ Hamiltonians [1214], each including a more or less detailed set of effects. Here we take the Hamiltonian constructed by Trebin et al [15] as a starting point. This eight-band $k \cdot p$ Hamiltonian is constructed by means of the invariant expansion of the Hamiltonian [14, 16, 17] and, when applied correctly, guarantees the inclusion of all matrix elements compatible with the $T_{d}$ symmetry group of the zinc-blendes up to the desired order in the electron wavevector $\boldsymbol{k}$. Due to the way that it has been constructed, this Hamiltonian takes into account all the effects of the spin-orbit interaction in the matrix elements up to $k^{2}$, and in particular the second-order s-p coupling via remote $\Gamma_{5}$ states [18] responsible for most of the contribution to the spin splitting in the conduction band (CB). Strain and coupled strain/spin-orbit effects are also properly described by this method.

After applying the usual EFA prescription [19-21] to the bulk Hamiltonian, the resulting system of coupled differential equations is solved by applying the discretization scheme to the differential operators described in Chuang and Chang [22]. From this follows a sparse eigenvalue problem that can be easily solved. The discretization scheme used provides us with a Hermitian discretized version of the Hamiltonian and also yields appropriate interface conditions naturally [23a]. A nice side effect of working with difference equations as opposed to differential equations-i.e., working in real space versus reciprocal space-is that the spurious solutions appearing in other methods $[24,25]$ are not present here. This is due to the bands along the growth (discretization) direction being periodic in $\boldsymbol{k}$-space and, therefore, not going out of control at large wavevectors. We have also developed methods to ensure that bulk bands from the discretized Hamiltonian do not enter the gap region [26].

With the help of computer software that automates algebraic operations [27], it is seen that the EFA Hamiltonian corresponding to structures with a symmetric sequence of layers and thicknesses transforms according to $\mathrm{D}_{2 \mathrm{~d}}$, while for asymmetric structures (i.e. asymmetric sequences) it transforms according to $\mathrm{C}_{2 \mathrm{v}}$. This is at variance with the majority of EFA implementations, which lack the inclusion of BIA effects and reproduce an approximate $\mathrm{D}_{4 \mathrm{~h}}$ symmetry [9] for symmetric structures ${ }^{4}$, not predicting any spin splitting. When necessary, the $\mathrm{C}_{2 \mathrm{v}}$ symmetry of a no-common-atom QW such as AlSb/InAs/AlSb can be reproduced in this EFA implementation by a proper rearrangement of the layer boundaries [23a]. The only case that cannot be modelled is when a common-atom heterostructure has a $\mathrm{C}_{2 \mathrm{v}}$ symmetry due to different bond orientation at the interfaces. The proper symmetry reproduction will allow the study of effects due to the reduced symmetry, such as the presence of optical anisotropy [28, 29], the mixing of heavy-hole and light-hole states on top of the valence band [8,30], and the spin splittings appearing in the band structure of zinc-blende heterostructures, which is shown in the rest of this paper.

\footnotetext{
4 Again, here 'symmetric' is meant to refer to the macroscopic arrangement of layers and their thicknesses.
}

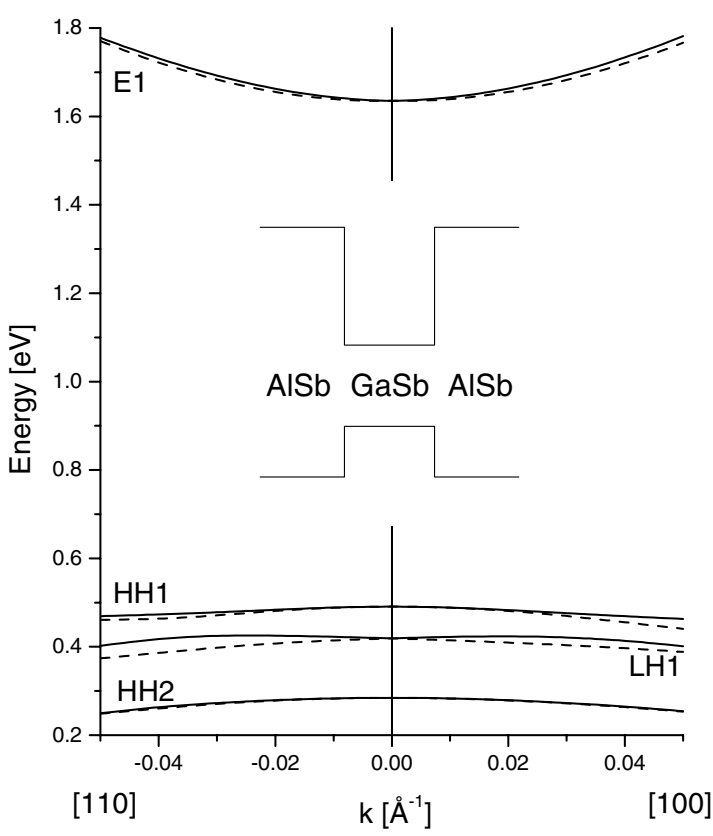

Figure 1. Bands along [100] and [110] for an $\mathrm{AlSb} / \mathrm{GaSb} / \mathrm{AlSb}$ SQW, 10 ML thick, with BIA terms. The inset shows the band diagram.

\section{Results and discussion}

Figure 1 shows the band structures along the [100] and the [110] directions of a common-atom AlSb/GaSb/AlSb symmetric quantum well (SQW), grown along the [001] direction and with a well thickness of 10 monolayers $(30.5 \AA)$, with the BIA terms included. The labels E1, HH1, LH1, and $\mathrm{HH} 2$ shown in the plots correspond to the first electron, first heavy-hole, first light-hole, and second heavy-hole states in the QW respectively. They refer to the main bulk state contribution at $k=0$. In agreement with group theory arguments, the bands are split along both directions except at the zone centre.

We have performed calculations for several directions and found that the splitting in the $\mathrm{CB}$ is isotropic and linear close to the $\Gamma$ point, with a proportionality coefficient of $\alpha_{B I A}=22 \times 10^{-10} \mathrm{eV} \mathrm{cm}$, where the splitting is

$$
\Delta_{B I A}=2 \alpha_{B I A} k
$$

Although this is an isotropic linear spin splitting, this must not be confused with the Rashba splitting [31]. Bychkov and Rashba introduced a splitting coefficient $\alpha_{R}$ in the context of asymmetric quantum wells (AQWs). The splitting studied there is derived from a model Hamiltonian that describes only SIA effects, but not BIA. As a consequence, the spin directions that they predict do not apply to the SQW situation [23a].

The computed $\alpha_{B I A}$ for this structure is about half of the predicted Rashba coefficient for some asymmetric structures $[26,32]$ where only SIA contributions are taken into account. This indicates that situations may arise where BIA effects need to be carefully studied before neglecting them in a calculation.

BIA also affects considerably the bands of AQWs. The interplay of SIA and BIA effects in AQWs adds a level of variety to the analysis of the behaviour of the spins in the $C B$. 
a)

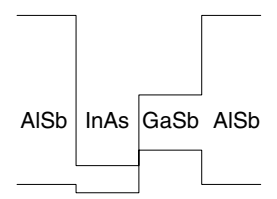

b) $\mathrm{AlSb} / \mathrm{lnAs} / \mathrm{GaSb} / \mathrm{AlSb} 8 / 8 / 8 / 8$ Superlattice

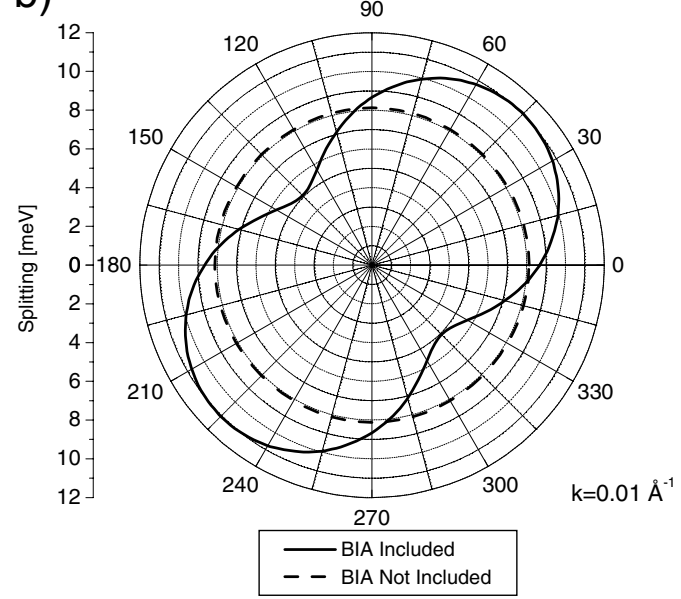

Figure 2. Angular dependence of the spin splitting in the $\mathrm{CB}$ of an $8 \mathrm{ML}$ AlSb/8 ML GaSb/8 ML InAs/8 ML AlSb superlattice. The splitting is calculated for $\boldsymbol{k}_{\|}=0.01(\cos \theta, \sin \theta) \AA^{-1}$. The distance of the line to the centre at $\theta$ represents the amount of splitting. The solid curve includes BIA effects, while the dashed curve does not.

However, the two-band model Hamiltonian given by Silva [33] keeps the analysis quite simple. For a [001] structure, the SIA and BIA contributions to the splitting can be described by the Hamiltonian $H_{I A}$ made from the addition of the BIA and the Rashba contributions:

$$
\begin{gathered}
H_{I A}=\alpha_{B I A}\left(\sigma_{x} k_{x}-\sigma_{y} k_{y}\right)+\alpha_{R}\left(\sigma_{x} k_{y}-\sigma_{y} k_{x}\right) \\
=\sigma_{x}\left(\alpha_{R} k_{y}+\alpha_{B I A} k_{x}\right)-\sigma_{y}\left(\alpha_{B I A} k_{y}+\alpha_{R} k_{x}\right),
\end{gathered}
$$

where $\alpha_{B I A}\left(\alpha_{R}\right)$ is the coefficient describing BIA (SIA) effects. From here, making an analogy with the Zeeman splitting, it is easy to find that the splitting in the CB close to the zone centre will be

$$
\Delta_{I A}=2 k \sqrt{\alpha_{R}^{2}+2 \alpha_{R} \alpha_{B I A} \sin 2 \theta+\alpha_{B I A}^{2}},
$$

where $\theta$ is the in-plane polar angle.

As expected, our eight-band model can reproduce the behaviour predicted by (3). The solid curve in figure 2(b) shows the angular dependence of the splitting for an $\mathrm{AlSb} / \mathrm{InAs} / \mathrm{GaSb} / \mathrm{AlSb} \mathrm{AQW}$ (shown in figure 2(a)). It corresponds to $\alpha_{S I A}=40.3 \times 10^{-10} \mathrm{eV} \mathrm{cm}$ and $\alpha_{B I A}=$ $15.0 \times 10^{-10} \mathrm{eV} \mathrm{cm}$. The dashed curve in figure 2 corresponds to the same calculation without the BIA effects included. The linear splitting with SIA only is isotropic. In this way the BIA effects are quantified, and it must be concluded that they must be taken into account for an accurate description of the bands in this structure. This is clearly so in the [110] direction, where the contributions are added linearly, but it is also true to a lesser degree in the [100] direction, where the contributions are added quadratically. This also has an impact on the theory of extraction of the Rashba coefficient from Shubnikov-de Haas
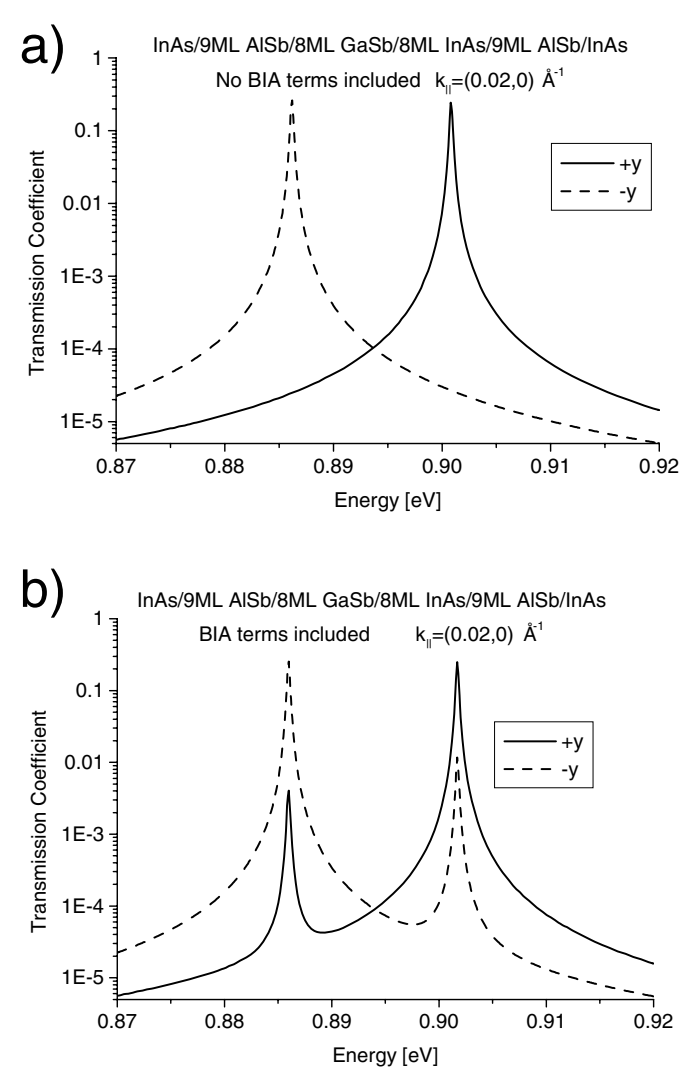

Figure 3. Tunnelling transmission coefficients for an InAs/9 ML AlSb/8 ML GaSb/8 ML InAs/9 ML AlSb/InAs asymmetric double barrier at zero bias. The electrons are taken to come with $\boldsymbol{k}_{\|}=(0.02,0) \AA^{-1}$. In plot (a), the Rashba effect splits the transmission peak into two for spins pointing along $+y$ (solid curve) and $-y$ (dashed curve). In plot (b), BIA effects are included and the direction of the resonant spins changes.

measurements in two-dimensional electron gases (2DEGs), where BIA effects have usually been neglected [34-36].

The EFA code for band structure computations can be adapted to calculate transmission coefficients in heterostructures by just setting the appropriate boundary conditions [37]. We have studied the effects of BIA on the transmission coefficients of an 9/8/8/9 ML AlSb/GaSb/InAs/AlSb double-barrier structure (see figure 2(a)) clad with InAs electrodes. Figure 3(a) shows the transmission coefficient for two different incoming spins with $\boldsymbol{k}_{\|}=(0.02,0) \AA^{-1}$ when BIA effects are not included. The position of the resonant peak is clearly dependent on the incoming spin. This is due to the fact that the Rashba effects splits the resonant states in the double barrier, and the incoming spins have been selected in such a way as to couple to only one of the quasibound resonances [38, 39]. There have been spin filter device proposals based on this effect $[39,40]$.

The effect of BIA is to change the spin of the barrier quasibound states [23a]. Now, if the incoming states are left unchanged, they will have a finite overlap with both of the new resonant spins, causing the presence of new transmission peaks, as shown in figure 2(b). In the particular structure shown in figure 2 it is seen that BIA has a small effect, but in general BIA will change the direction along which the filtered spins must be analysed to achieve maximum filtering efficiency. 


\section{Acknowledgments}

The authors would like to thank D L Smith and J N Schulman for helpful discussions. This work was supported by a subcontract with HRL, LLC in conjunction with the Office of Naval Research under contract No MDA972-01-C-0002. Part of the work described in this paper was carried out at the Jet Propulsion Laboratory, California Institute of Technology, and was sponsored by the Defense Advanced Research Projects Agency's Spins in Semiconductors (SpinS) programme.

\section{References}

[1] Wolf S A 2000 J. Supercond. 13195

[2] Datta S and Das B 1990 Appl. Phys. Lett. 56665

[3] Fiederling R, Keim M, Reuscher G, Ossau W, Schmidt G, Waag A and Molenkamp L W 1999 Nature 402787

[4] Ting D Z-Y, Cartoixà X, Chow D H, Moon J S, Smith D L, McGill T C and Schulman J N 2002 IEEE Trans. Magn. at press

[5] Lommer G, Malcher F and Rössler U 1988 Phys. Rev. Lett. 60728

[6] Cardona M, Christensen N E and Fasol G 1988 Phys. Rev. B 381806

[7] Dresselhaus G 1955 Phys. Rev. 100580

[8] Magri R and Zunger A 2000 Phys. Rev. B 6210364

[9] Tronc P and Kitaev Y E 2001 Phys. Rev. B 63

[10] Winkler R and Rössler U 1993 Phys. Rev. B 488918

[11] Wissinger L, Rössler U, Winkler R, Jusserand B and Richards D 1998 Phys. Rev. B 5815375

[12] Kane E O 1957 J. Phys. Chem. Solids 1249

[13] Pidgeon C R and Brown R N 1966 Phys. Rev. 146575

[14] Bir G L and Pikus G E 1974 Symmetry and Strain-Induced Effects in Semiconductors 1st edn (New York: Wiley)

[15] Trebin H-R, Rössler U and Ranvaud R 1979 Phys. Rev. B 20686

[16] Pikus G E 1961 Zh. Eksp. Teor. Fiz. 41 1258-73 Pikus G E 1962 Sov. Phys.-JETP 14 898-907

[17] Pikus G E 1961 Zh. Eksp. Teor. Fiz. 41 1507-21 Pikus G E 1962 Sov. Phys.-JETP 14 1075-85
[18] Kane E O 1966 Semiconductors and Semimetals vol 1, ed R $\mathrm{K}$ Willardson and A C Beer (New York: Academic) pp 75-100

[19] Luttinger J M and Kohn W 1955 Phys. Rev. 97869

[20] Bastard G 1981 Phys. Rev. B 245693

[21] Bastard G 1982 Phys. Rev. B 257584

[22] Chuang S L and Chang C S 1997 Semicond. Sci. Technol. 12252

[23] Cartoixà X, Ting D Z-Y and McGill T C 2002 Phys. Rev. B submitted

Cartoixà X, Ting D Z-Y and McGill T C 2002 Preprint cond-mat/0212394

[24] Schuurmans M F H and 't Hooft G W 1985 Phys. Rev. B 318041

[25] Meney A T, Gonul B and O’Reilly E P 1994 Phys. Rev. B 5010893

[26] Cartoixà X, Ting D Z-Y and McGill T C 2002 J. Comput. Electron. 1141

[27] Wolfram Research, Inc. 1999 Mathematica version 4 (Champaign, IL: Wolfram Research, Inc.)

[28] Krebs O and Voisin P 1996 Phys. Rev. Lett. 771829

[29] Magri R and Ossicini S 2001 Phys. Rev. B 63

[30] Ivchenko E L, Kaminski A Y and Rössler U 1996 Phys. Rev. B 545852

[31] Bychkov Y A and Rashba E I 1984 J. Phys. C: Solid State Phys. 176039

[32] Cartoixà X, Ting D Z-Y, Daniel E S and McGill T C 2001 Superlatt. Microstruct. 30309

[33] Silva E A D E 1992 Phys. Rev. B 461921

[34] Luo J, Munekata H, Fang F F and Stiles P J 1988 Phys. Rev. B 3810142

[35] Luo J, Munekata H, Fang F F and Stiles P J 1990 Phys. Rev. B 417685

[36] Schäpers T, Engels G, Lange J, Klocke T, Hollfelder M and Lüth H 1998 J. Appl. Phys. 834324

[37] Liu Y X, Ting D Z-Y and McGill T C 1996 Phys. Rev. B 54 5675

[38] Silva E A D E and LaRocca G C 1999 Phys. Rev. B 59 R 15583

[39] Voskoboynikov A, Lin S S, Lee C P and Tretyak O 2000 J. Appl. Phys. 87387

[40] Koga T, Nitta J, Takayanagi H and Datta S 2002 Phys. Rev Lett. 88126601 